\title{
Forecasting the Tuberculosis Incidence Using a Novel Ensemble Empirical Mode Decomposition-Based Data-Driven Hybrid Model in Tibet, China
}

\author{
Jizhen $\mathrm{Li}^{\prime}$ \\ Yuhong $\mathrm{Li}^{2}$ \\ Ming $\mathrm{Ye}^{3}$ \\ Sanqiao Yao' \\ Chongchong $\mathrm{Yu}^{\prime}$ \\ Lei Wang ${ }^{4}$ \\ Weidong $\mathrm{Wu}$ (D) $^{\prime}$ \\ Yongbin Wang
}

'Department of Epidemiology and Health Statistics, School of Public Health, Xinxiang Medical University, Xinxiang, Henan Province, People's Republic of China; ${ }^{2}$ National Center for Tuberculosis Control and Prevention, China Center for Disease Control and Prevention, Beijing, People's Republic of China; ${ }^{3}$ Preventive Medicine Clinic, Xinxiang Center for Disease

Control and Prevention, Xinxiang, Henan Province, People's Republic of China; ${ }^{4}$ Center for Musculoskeletal Surgery, Charité-Universitätsmedizin Berlin, Corporate Member of Freie Universität Berlin, Humboldt-Universität Zu Berlin and Berlin Institute of Health, Berlin, Germany
Correspondence: Yongbin Wang Department of Epidemiology and Health Statistics, School of Public Health, Xinxiang Medical University, Xinxiang, Henan Province, 453000, People's Republic of China

Email wybwho@163.com
Objective: The purpose of this study is to develop a novel data-driven hybrid model by fusing ensemble empirical mode decomposition (EEMD), seasonal autoregressive integrated moving average (SARIMA), with nonlinear autoregressive artificial neural network (NARNN), called EEMD-ARIMA-NARNN model, to assess and forecast the epidemic patterns of TB in Tibet.

Methods: The TB incidence from January 2006 to December 2017 was obtained, and then the time series was partitioned into training subsamples (from January 2006 to December 2016) and testing subsamples (from January to December 2017). Among them, the training set was used to develop the EEMD-SARIMA-NARNN combined model, whereas the testing set was used to validate the forecasting performance of the model. Whilst the forecasting accuracy level of this novel method was compared with the basic SARIMA model, basic NARNN model, error-trend-seasonal (ETS) model, and traditional SARIMA-NARNN mixture model.

Results: By comparing the accuracy level of the forecasting measurements including rootmean-square error, mean absolute deviation, mean error rate, mean absolute percentage error, and root-mean-square percentage error, it was shown that the EEMD-SARIMA-NARNN combined method produced lower error rates than the others. The descriptive statistics suggested that TB was a seasonal disease, peaking in late winter and early spring and a trough in autumn and early winter, and the TB epidemic indicated a drastic increase by a factor of 1.7 from 2006 to 2017 in Tibet, with average annual percentage change of 5.8 (95\% confidence intervals: $3.5-8.1$ ).

Conclusion: This novel data-driven hybrid method can better consider both linear and nonlinear components in the TB incidence than the others used in this study, which is of great help to estimate and forecast the future epidemic trends of TB in Tibet. Besides, under present trends, strict precautionary measures are required to reduce the spread of TB in Tibet.

Keywords: tuberculosis, incidence rate, time series analysis, statistical models, forecasting

\section{Introduction}

Tuberculosis (TB) is a chronic communicable disease caused by the bacillus Mycobacterium tuberculosis, it is a major cause of ill-health, ranking among the 10 leading causes of morbidity and mortality globally. ${ }^{1}$ The WHO estimated that about 10.0 million people developed TB in 2019 around the globe. ${ }^{2}$ Furthermore, there were an estimated 1.4 million deaths associated with TB in 2019, leading to an annual approximately $1.8 \%$ and $2.8 \%$ reductions between 2015 and 2019 in the 
incidence and death rates, respectively, worldwide. ${ }^{2}$ Currently, the global case notifications were mainly reported from 30 high TB burden countries, accounting for around $87 \%$ of the worldwide totals. ${ }^{2}$ Of them, ranking the third is China, where the proportion of the reported cases reaches $8.4 \%$ in the world. ${ }^{2}$ Moreover, China also has the second share (14\%) of the global burden of drugresistant TB in 2019, despite progress in cutting TB incidence with an average annual percent change of $3.2 \%$ between 1990 and 2017. ${ }^{3}$ However, recent years have been witnessing a risk of resurgence in the TB incidence in some regions in China, attributable to climate change, alcohol use disorders, diabetes, HIV infection, smoking, and undernourishment, which have suppressed the progress of the end TB strategy with a target of $90 \%$ and 95\% reductions in the TB incidence and death rates, respectively, in the period 2015-2035. ${ }^{1,2,4,5}$ To accelerate progress towards such an ambitious goal, accurately estimating and forecasting the past, current, and future epidemic trends of TB play a pivotal role in forming the targeted prevention and control measures and in adjusting the present interventions.

There is a large volume of studies forecasting the epidemiological trends of communicable diseases using different statistical techniques, such as the seasonal autoregressive integrated moving average (SARIMA) method, ${ }^{6}$ exponential smoothing method, ${ }^{7}$ generalized regression neural network (GRNN) method, ${ }^{8}$ nonlinear autoregressive neural network (NARNN) method, ${ }^{9}$ backpropagation neural network (BPNN) method, ${ }^{1}$ multivariate linear regression method, ${ }^{10}$ and Elman and Jordan recurrent neural networks. ${ }^{11}$ Among them, the most frequently used linear method is the SARIMA model, ${ }^{12-15}$ whereas the nonlinear method is the NARNN model. ${ }^{5,9,12,16}$ However, there are various factors affecting and limiting the epidemiological patterns of diseases. ${ }^{1,17,18}$ For this reason, the epidemics of communicable diseases are often characterized by linear and nonlinear patterns. ${ }^{19}$ However, the above-mentioned statistical techniques are often insufficient to capture either tendencies or randomness in the time series, which may limit the generalization of the findings from the mentioned models. Therefore, to improve the prediction accuracy and reliability, an alternative approach is expected to be customized for use with both the above two traits included in the object series. Inspired by such an idea, many studies have adopted a hybrid technique comprising a linear model and a nonlinear model to yield more accurate prediction by integrating each model's superiority., ${ }^{9,20,21}$

Over the last years, wavelet analysis has become a popular method capable of decomposing the data into different high-scale and low-frequency components (linear trait) and low-scale and high-frequency components (nonlinear trait) particularly when target series shows complex nonstationary and nonlinear characteristics. $^{22}$ More recently, a new wavelet analysis approach-ensemble empirical mode decomposition (EEMD) was developed by adding noise to overcome the mode mixing disadvantage of the traditional empirical mode decomposition (EMD). ${ }^{23}$ Importantly, different from the most commonly used discrete wavelet transform techniques that may generate different decomposition results since they are required to predetermine basis functions, ${ }^{24}$ EEMD is a self-adaptive, intuitive, direct, and empirical decomposition method for the nonstationary and nonlinear time series. ${ }^{23}$ Considering the superiority of the EEMD technique in decomposing the data and the worsening epidemic trends of TB in Tibet with the highest TB morbidity in China in the past years, ${ }^{25}$ we thus proposed a novel forecasting method (designated as EEMD-SARIMA-NARNN hybrid method) by integrating the SARIMA and NARNN models based on the EEMD technique to capture both the tendencies and randomness in the time series simultaneously. Specifically, the EEMD technique was first applied to decompose the TB incidence series into several Intrinsic Mode Functions (IMFs) components representing the different nonlinear parts of the data and a residue component denoting the tendency of the data. Second, using the NARNN and SARIMA methods to model the IMFs and residue components, respectively. Finally, the forecasting values from the IMFs and residue components were aggregated as the predicted results from the EEMD-SARIMA-NARNN hybrid technique. By doing so, the linear and nonlinear parts contained in the TB incidence series can be considered by this data-driven mixture method simultaneously. Whilst we also compared the fitted and predicted results from the basic SARIMA model, basic NARNN model, and the traditional SARIMA-NARNN mixture model with that from our proposed novel hybrid method to judge the forecasting performance of this novel data-driven method. 


\section{Materials and Methods}

\section{Study Area and Data Source}

Tibet is located in the southwest of the People's Republic of China. It is one of the five ethnic autonomous regions in China, and its average elevation is above $4000 \mathrm{~m}$, known as the "roof of the world". Tibet covers $1228.4 \mathrm{~km}^{2}$. At the end of 2019, the resident population was 3.506 million.

The monthly incident cases between January 2006 and December 2017 reported to the Center for Disease Control and Prevention (CDC) were taken from the National Notifiable Infectious Disease Surveillance System (NNIDSS), and the population data during the same period from the Bureau of statistics of Tibet Autonomous Region (http://tjj.xizang.gov.cn/). A total of 144 observations across 12 years were aggregated. Generally, it is required to use preferably 100 observations or more to develop object models so that a reliable and stable result is obtained. $^{26}$ Therefore, the observations used in this research were partitioned into two parts including a training dataset comprising 132 observations (from January 2006 to December 2016) and a testing dataset comprising 12 observations (from January to December 2017).

\section{Ethics Approval and Consent to Participate}

The study protocol was approved by the study institutional review board of the Xinxiang Medical University (No: XYLL-2019,072), and consent is not needed for this study since we only collected the reported monthly TB cases publicly available without the detailed individual information.

\section{A New Hybrid Model of EEMD-SARIMA- NARNN \\ EEMD Technique}

In the past, albeit EMD technique shows remarkable superiority in signal analysis, particularly for the data produced in noisy nonlinear and non-stationary processes, there are two major weaknesses including the edge-effects and mode-mixing, ${ }^{20,23,27}$ especially for the mode-mixing disadvantage, which not only results in the mixing of different scale vibration modes but can even cause the loss of the physical meaning of each IMFs. ${ }^{28}$ The EEMD method was thus developed based on the EMD algorithm to overcome the barriers of the original technique. ${ }^{23}$ In the EEMD approach, it uses a powerful method of ensemble mean to improve the accuracy of measurements. ${ }^{23}$ Namely, it defines the actual individual IMFs components as the average of an ensemble of experiments, each IMF component is composed of the signal and noise of finiteamplitude. ${ }^{20}$ The decomposition procedure of the EEMD approach can be in the form as below.

Initially, add white noise $\mathrm{w}(t)$ to the given time series $x$ $(t)$, and the obtained new series can be expressed as

$$
X(t)=x(t)+w(t)
$$

Secondly, decompose the produced series into IMFs with the help of the EMD algorithm.

Thirdly, repeat the above two steps using various white noise series, the resulting results were added to the original series each time.

Finally, average the ensemble of the corresponding IMFs generated by the EMD decomposition technique.

In the process of decomposition, the numbers of the ensemble and the amplitude of the added white noise play a pivotal role in the obtained final results. ${ }^{29}$ Fortunately, a well-demonstrated statistical rule helps to determine these two key parameters ${ }^{23}$ :

$$
\varepsilon_{n}=\frac{\varepsilon}{N}
$$

where $N$ represents the numbers of the ensemble, $\varepsilon$ is the amplitude of the added white noise, and $\varepsilon_{n}$ signifies the standard deviation of the error. Prior studies have found that the parameter settings with the ensemble numbers of 100 and the amplitude of added white noise of 0.2 times standard deviation can generate a satisfactory result. ${ }^{23,30}$

\section{EEMD-SARIMA-NARNN Hybrid Model}

To this end that makes full use of the possible linear and nonlinear information contained in the target series, inspired by the idea of "decomposition and ensemble" of the EEMD technique and the powerful ability to extract nonlinear information of the NARNN model, $^{31}$ the EEMD-SARIMA-NARNN hybrid model was thus proposed (Figure S1). In this project, the time series of TB morbidity was decomposed into different IMFs subseries and residue terms. Then, each of IMFs subseries was predicted using a suitable NARNN model, while the residue term was forecasted with an appropriate SARIMA model. As such, the final results can be derived by integrating the resulting values of the SARIMA model with the counterparts of the NARNN approaches. By doing so, the hybrid model can then attain a capacity that unearths 
all possible components included in the data. The specified equation of a SARIMA-NARNN combined model can be written as

$$
\begin{gathered}
I M F_{t}=\sum_{i=1}^{N}\left(f \left(I M F(t-1), \cdots, I M F_{1}(t-d)+\Lambda\right.\right. \\
+\left(f \left(I M F_{N}(t-1), \cdots, I M F_{N}(t-d)\right.\right. \\
\hat{y}=\hat{a}+I M F_{t}
\end{gathered}
$$

where $y$ represents the estimates using the proposed hybrid method, $\hat{a}$ is the estimates of residue terms using the SARIMA method, $\hat{e}$ refers to the estimates of IMFs subseries using the NARNN method.

Furthermore, the SARIMA method, NARNN method, and traditional SARIMA-NARNN method were created according to the steps described in supplementary material.

\section{Statistical Analysis}

The TB epidemic trends and changes were measured with average annual percentage change (AAPC) based on the joinpoint regression program (Version 4.8.0.1). The basic SARIMA method was constructed with $\mathrm{R}$ software (version 3.4.3, R Development Core Team, Vienna, Austria), and the EEMD, basic NARNN, SARIMA-NARNN, and EEMD-SARIMA-NARNN methods were developed using MATLAB software (Version R2014a, MathWorks, Natick, MA, USA). The Lagrangian Multiplier (LM) method was used to test the conditional heteroskedastic behavior and volatility (ARCH effect) of the residual series from the selected four models. ${ }^{32}$ The root-mean-square error (RMSE), mean absolute deviation (MAD), mean error rate (MER), mean absolute percentage error (MAPE), and root-mean-square percentage error (RMSPE) were chosen to assess the predictive accuracy levels among models. The lower the indices' values are, the better the models are.

$$
\begin{aligned}
\operatorname{RMSE} & =\sqrt{\frac{1}{N}} \sum_{i=1}^{N}\left(X_{i}-\hat{\dot{i}}\right)^{2} \\
\operatorname{MAD} & =\frac{1}{N} \sum_{i=1}^{N}\left|X_{i}-\hat{X}\right| \\
\operatorname{MER} & =\frac{1}{N} \sum_{i=1}^{N} \mid \frac{X_{i}-\hat{X} \mid}{\overline{X_{i}}}
\end{aligned}
$$

$$
\begin{gathered}
\text { MAPE }=\frac{1}{\mathrm{~N}} \sum_{\mathrm{i}=1}^{\mathrm{N}} \frac{\left|\mathrm{X}_{\mathrm{i}}-\hat{\mathrm{X}}_{\mathrm{i}}\right|}{\mathrm{X}_{\mathrm{i}}} \times 100 \\
\text { RMSPE }=\sqrt{\frac{1}{\mathrm{~N}}} \sum_{\mathrm{i}=1}^{\mathrm{N}} \frac{\left(\mathrm{X}_{\mathrm{i}}-\hat{\mathrm{X}}_{\mathrm{i}}\right)^{2}}{\mathrm{X}_{\mathrm{i}}}
\end{gathered}
$$

where $X_{i}$ signifies the observed TB values, $X_{i}$ is the estimates using the chosen approaches, $\overline{X_{i}}$ refers to the mean of all the observed TB values, and $N$ stands for the number of simulations and forecasts.

\section{Results}

\section{Descriptive Analysis}

During the period 2006-2017, the reported TB cases totaled 45,025 with notified 3753 cases annually, leading to annualized and monthly incidence rates of 121.9 and 10.2 per 100,000 persons, respectively. As illustrated in Figure S2, when the seasonal-trend decomposition procedure based on Loess (STL) technique was applied to the TB morbidity series, it was found that the TB epidemic showed a drastic increase by a factor of 1.7 from 2006 to 2017, with AAPC $=5.8$ (95\% confidence intervals: $3.5-8.1$; $\mathrm{Z}=5.8, P<0.001$ ) (Figure S3). The highest TB morbidity rate was noted in 2017 (152.0 cases per 100,000 people), it has increased 2.4-fold compared with 2008 when the morbidity rate was lowest (64.7 cases per 100,000 people). Besides, it was observed that the TB morbidity had notable seasonal effects, with a peak occurred in January until August, especially in January and March, and a trough in September until December, particularly in December (Figures S2 and $\underline{\mathrm{S} 4}$ ).

\section{The Best-Fitting SARIMA Method}

The original TB incidence series showed a unit root $(\mathrm{ADF}=$ $-1.3, P=0.2$ ) and marked seasonal effects (Figure S2), indicating a nonstationary series, and thus seasonally differencing once to remove the seasonal variation of the data. By doing so, the differenced data met the prerequisite of the SARIMA model development, being stationary $(\mathrm{ADF}=-3.4$, $P=0.001$ ). Afterward, by plotting the ACF and PACF graphs for the stationary series, the autocorrelations at lags 1,2, and 3 and partial autocorrelations at lags 1 and 2 were found to touch the significance levels (Figure S5), and we observed that the values of the key parameters (p, q, P, and Q) of SARIMA model exceeded 2, the model's parameter suggested no statistical significance, and thereby we selected 
several plausible models with the key parameters were between 0 and 2 . Table 1 demonstrates that the $\operatorname{SARIMA}(2,0,0)(2,1,0)_{12}$ model seems to provide adequate modeling for the object series since this model presented the lowest values of performance indices ( $\mathrm{AIC}=409.2$, $\mathrm{CAIC}=409.7$, and $\mathrm{BIC}=423.2$ ) among the possible nine models, and statistical test of the estimated parameters for this method was indicated significantly different (Table 2). Moreover, both the Ljung-Box Q test and LM test showed a $P$-value greater than 0.05 (Figure $1 \mathrm{~A}$, Tables $\mathrm{S} 1$ and $\underline{\mathrm{S} 2}$ ), suggesting that the residuals behaved like white noise and the ARCH effect had been removed from the residuals. The above tests mean that the $\operatorname{SARIMA}(2,0,0)(2,1,0)_{12}$ model is appropriate for describing this time series. Therefore, this model is taken as the best.

\section{The Best-Fitting NARNN Method}

As described in previous studies, the ANNs models with hidden units between 10 and 20 and feedback delays between 3 and 7 can often provide a satisfactory prediction and ease overfitting to a certain degree. ${ }^{33-37}$ Therefore, we trained a series of networks with the mentioned-above ranges of hidden units and feedback delays by trial and error so that a preferred model was discovered. By doing so, the NARNN $(16,6)$ model with 16 hidden units and 6 feedback delays tended to be indicated the optimal in that this network structure produced the lowest MSE values in the training (0.6),

Table I The Identified Nine Plausible SARIMA Methods and Their Information Criteria

\begin{tabular}{|l|l|l|l|l|}
\hline Models & AIC & CAIC & BIC & $\begin{array}{l}\text { Log- } \\
\text { Likelihood }\end{array}$ \\
\hline SARIMA $(2,0,0)(2, I, 0)_{12}$ & 409.22 & 409.74 & 423.16 & -199.61 \\
\hline SARIMA $(I, 0,2)(2, I, 0)_{12}$ & 410.01 & 410.75 & 426.73 & -199.00 \\
\hline SARIMA $(I, 0, I)(2, I, 0)_{12}$ & 411.83 & 412.36 & 425.77 & -200.92 \\
\hline SARIMA $(0,0,2)(2, I, 0)_{12}$ & 440.42 & 440.94 & 454.35 & -215.21 \\
\hline SARIMA $(2,0, I)(2, I, 0)_{12}$ & 410.70 & 411.44 & 427.42 & -199.35 \\
\hline SARIMA $(2,0, I)(0, I, I)_{12}$ & 415.85 & 416.37 & 429.78 & -202.92 \\
\hline SARIMA $(2,0, I)(0, I, 2)_{12}$ & 416.17 & 416.91 & 432.90 & -202.09 \\
\hline SARIMA $(2,0,0)(I, I, I)_{12}$ & 415.06 & 415.59 & 429.00 & -202.53 \\
\hline SARIMA $(2,0,0)(I, I, 0)_{12}$ & 432.80 & 433.14 & 443.95 & -212.40 \\
\hline
\end{tabular}

Abbreviations: SARIMA, seasonal autoregressive integrated moving average method; AIC, Akaike's Information Criterion; BIC, Bayesian Information Criterion.
Table 2 Statistical Test of the Estimated Parameters for the Optimal SARIMA $(2,0,0)(2,1,0)_{12}$ Method

\begin{tabular}{|l|l|l|l|l|}
\hline Variables & Estimates & Standard Error & $\boldsymbol{t}$ & $\boldsymbol{P}$ \\
\hline ARI & 0.519 & 0.089 & 5.831 & $<0.001$ \\
\hline AR2 & 0.329 & 0.092 & 3.576 & $<0.001$ \\
\hline SARI & -0.736 & 0.086 & -8.558 & $<0.001$ \\
\hline SAR2 & -0.526 & 0.089 & -5.910 & $<0.001$ \\
\hline
\end{tabular}

Abbreviations: SARIMA, seasonal autoregressive integrated moving average method; ARI, autoregressive method at lag I; AR2, autoregressive method at lag 2; SARI, seasonal autoregressive method at lag I; SAR2, seasonal autoregressive method at lag 2 .

validation (4.5), and testing (3.8), and all $\mathrm{R}$ values were greater than 0.8 in the training (1.0), validation (0.8), testing (0.8), and the whole data (0.9) (Table 3 and Figure S6). Also, as shown in Figure S7, the modeling results of this model agreed pretty well with the observed values as most of the errors were between -1 and 1. Additionally, the Ljung-Box $\mathrm{Q}$ and LM-test statistics for the residual sequence yielded by this model showed a $P$-value greater than 0.05 (Tables S1 and $\underline{\mathrm{S}}$ ), and the sample autocorrelation and partial autocorrelation for the in-sample forecast errors at different lags failed to exceed the significance bounds (Figure 1B), implying that the in-sample forecast errors showed a white noise and no ARCH effect. Thus, we are confident that the NARNN $(16,6)$ model was considered optimal in this study.

\section{The Best-Fitting Traditional SARIMA-NARNN Mixture Method}

In the process of constructing the traditional SARIMANARNN method, the residual series from the SARIMA model was utilized to train the best NARNN model. Similarly, we found that the NARNN $(19,6)$ method with 19 hidden units and 6 feedback delays appeared to be abundant for modeling the object series via repeated modeling as this model had a relatively great $\mathrm{R}$-value and a relatively low MSR value in the training, validation, and testing data (Table 3). Further, we investigated the residual series from the $\operatorname{SARIMA}(2,0,0)(2,1,0)_{12}$-NARNN $(16,6)$ mixture model using the Ljung-Box Q and LM tests, showing that this residual series belonged to a white noise series and also no ARCH effect was noted in the residuals (Tables $\underline{\mathrm{S} 1}$ and $\underline{\mathrm{S}}$ ). Besides, the sample autocorrelations and partial autocorrelations for the in-sample forecast errors inside the 95\% uncertainty bounds also indicated that the errors met 

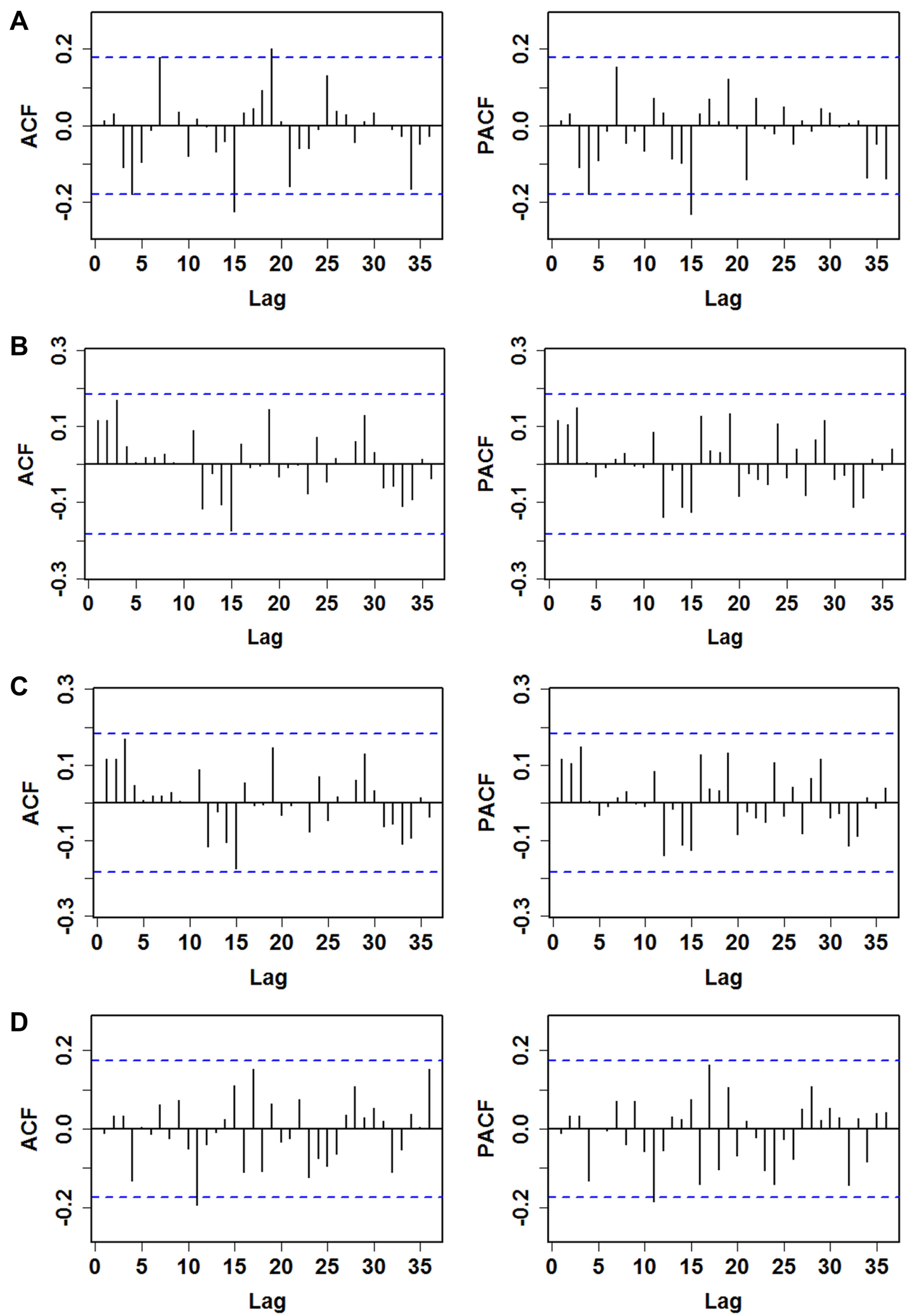

Figure I Estimated autocorrelogram and partial autocorrelogram of the residual sequence to forecast the TB epidemic patterns using (A) SARIMA model, (B) basic NARNN model, (C) traditional SARIMA-NARNN hybrid model, and (D) novel EEMD-SARIMA-NARNN hybrid model. As seen, the estimated autocorrelations and partial autocorrelations of the errors from the different models almost fell into the $95 \%$ confidence intervals (Cls) with few exceptions, such as the autocorrelations at lags 15 and 19 and partial autocorrelations at lags 15 in (A), and autocorrelations and partial autocorrelations at lag 11 in (D), these are also reasonable because higher-order autocorrelations and partial autocorrelations easily exceed the $95 \mathrm{Cls}$ by chance. 
Table 3 The Estimated Parameters of the Optimal NARNN Models for the Original Series and Decomposed Series Using the EEMD Method

\begin{tabular}{|l|c|c|c|c|c|c|c|c|c|}
\hline \multirow{2}{*}{$\begin{array}{l}\text { Target } \\
\text { Components }\end{array}$} & Hidden Units & \multirow{2}{*}{ Delays } & \multicolumn{3}{|c|}{ MSE } & & \multicolumn{3}{c|}{ R } \\
\cline { 4 - 9 } & & & Training & Validation & Testing & Training & Validation & Testing & Overall \\
\hline Original series & 16 & 6 & 0.648 & 4.531 & 3.760 & 0.973 & 0.842 & 0.848 & 0.944 \\
\hline $\begin{array}{l}\text { Residuals of } \\
\text { SARIMA model }\end{array}$ & 19 & 6 & 0.537 & 8.110 & 2.287 & 0.806 & 0.714 & 0.607 & 0.715 \\
\hline IMFI & 17 & 5 & 0.124 & 0.885 & 1.806 & 0.967 & 0.799 & 0.827 & 0.911 \\
\hline IMF2 & 16 & 5 & 0.052 & 0.101 & 0.226 & 0.992 & 0.979 & 0.971 & 0.988 \\
\hline IMF3 & 15 & 5 & 0.001 & 0.001 & 0.002 & 1.000 & 1.000 & 1.000 & 1.000 \\
\hline IMF4 & 16 & 6 & 0.000 & 0.000 & 0.000 & 1.000 & 1.000 & 1.000 & 1.000 \\
\hline IMF5 & 14 & 4 & 0.000 & 0.000 & 0.000 & 1.000 & 1.000 & 1.000 & 1.000 \\
\hline IMF6 & 14 & 5 & 0.000 & 0.000 & 0.000 & 1.000 & 1.000 & 1.000 & 1.000 \\
\hline
\end{tabular}

Abbreviations: SARIMA, seasonal autoregressive integrated moving average method; NARNN, nonlinear autoregressive artificial neural network.

the assumption of uncorrelatedness (Figure 1C). Thereby, the $\operatorname{SARIMA}(2,0,0)(2,1,0)_{12}$-NARNN $(16,6)$ mixture model is considered the best-performing in this work.

\section{The Best-Fitting Novel EEMD-SARIMA- NARNN Mixture Method}

The EEMD technique was employed to decompose the TB morbidity series. As listed in Figure 2, the original series was decomposed into six different IMFs subseries and a residue term. Subsequently, the residue term was modeled using the SARIMA method and the IMFs terms were modeled with different NARNN methods, and the resulting results showed that the SARIMA $(1,2,0)(1,0,1)_{12}$ method was the most suitable for modeling the residue term and NARNN(17, 5), NARNN(16, 5), NARNN $(15,5), \operatorname{NARNN}(16,6), \operatorname{NARNN}(14,4)$, and NARNN $(14,5)$ models were taken to be the best methods for modeling the IMF1 IMF6 subseries (Tables 3-4), respectively, and the diagnostic test results for the best SARIMA model and different NARNN models are shown in Figure 3 and S8-S14. Further checking results for the residuals from the new EEMD-SARIMA-NARNN mixture method are given in Tables S1-S2 and Figure 1D. The $P$-values for LM and Ljung-Box Q tests were greater than 0.05 (Tables

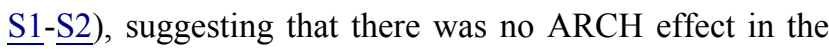
errors and there was little evidence of non-zero autocorrelations in the in-sample forecast errors at various lags, which could also be verified by the ACF and PACF plots as the sample autocorrelations and partial autocorrelations at different lags almost fell into the $95 \%$ significance bounds (Figure 1D). The above results intimated that this new data-driven mixture model can capture the short-term dependencies of the data and is adequate for forecasting the data.

\section{Assessing the Predictive Accuracy Levels Among Models}

We used the preferred individual models to predict the testing data, and then comparing the predictive accuracy levels among models from two aspects of the training and forecasting horizons based on five measures including RMSE, MAD, MER, MAPE, and RMSPE. The comparative results showed that our proposed EEMD-SARIMANARNN mixture model produced lower values of the above five measures compared with other methods used in this study both in training and forecasting horizons (Table 5). Likewise, visible in Figure 4, it makes sense that this new mixture technique can better capture the temporal dependency structure of the TB incidence series than the others.

\section{Discussion}

Accurately estimating the epidemics of infectious diseases acts as a basis for the decision-making process and the rational allocation of limited health resources under dynamic demand. ${ }^{1}$ The importance and originality of this 

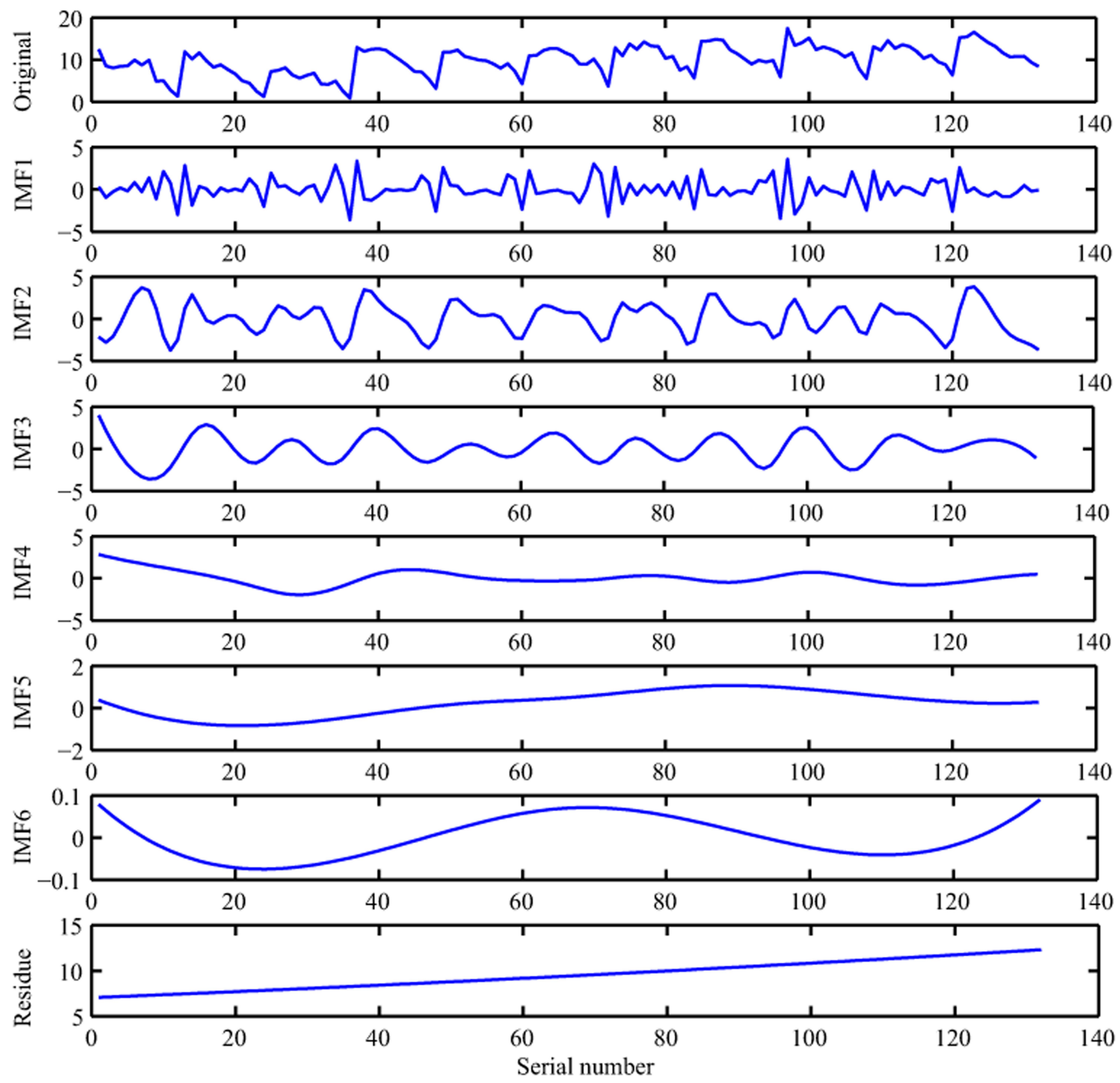

Figure 2 Intrinsic mode functions (IMFs) and residue components of the original TB incidence series using the EEMD.

work are that it creates a novel and more effective forecasting model for TB incidence series prediction by integrating the linear characteristics of a SARIMA method and nonlinear characteristics of the NARNN model based on the EEMD technique. By comparing the performance indicators of this novel model comprising RMSE, MAD, MER, MAPE, and RMSPE with the basic SARIMA model, basic NARNN model, and the traditional SARIMA-NARNN mixture model, it was observed that this new data-driven hybrid model showed better superiority than the others both in the fitted and predicted aspects. Often, the MAPE value is considered a useful measure to assess the forecasting accuracy of a model, with a value less than $10 \%$ indicating a highly accurate forecast, and a value less than $20 \%$ suggesting a good approximation to the observed values. ${ }^{38}$ Exhilaratingly, our proposed novel hybrid technique provided a highly accurate forecast for the TB morbidity series as the MAPE showed a value less than $10 \%$ both in the simulated and predicted horizons, which further reinforces the usefulness of our proposed new method as a helpful forecasting tool. Also, we used the TB incidence data in Qinghai 
Table 4 Statistical Test of the Estimated Parameters for the Optimal SARIMA $(1,2,0)(1,0,1)_{12}$ Method

\begin{tabular}{|l|l|l|l|l|}
\hline Variables & Estimates & Standard Error & $\boldsymbol{t}$ & $\boldsymbol{p}$ \\
\hline ARI & 1.000 & 0.002 & 578.647 & $<0.00 \mathrm{I}$ \\
\hline SARI & 0.984 & 0.029 & 34.493 & $<0.001$ \\
\hline SMAI & 0.692 & 0.152 & 4.564 & $<0.001$ \\
\hline
\end{tabular}

Abbreviations: SARIMA, seasonal autoregressive integrated moving average method; ARI, autoregressive method at lag I; SARI, seasonal autoregressive method at lag I; SMAI, seasonal moving average method at lag I.

province to provide an external validation for the predictive ability of our proposed hybrid method, ${ }^{12}$ Tables S3-S5 and Figures S15-S16 exhibit the resulting results, suggesting a same finding as our current work that the EEMDSARIMA-NARNN mixture method produces more accurate approximation to the TB incidence data in Qinghai. Besides, recent work of our research group indicates that the error-trend-seasonal (ETS) model can also accurately estimate and forecast the epidemic patterns of TB incidence, ${ }^{4}$ and thus we further compared the mimic and predicted abilities between our proposed hybrid technique and ETS model. The modeling results displayed that the ETS(A,AD,A) model is supposed to be considered the preferred (Tables S6-S7). Similarly, visible in Table S8, the EEMD-SARIMA-NARNN hybrid technique also significantly outperforms the optimal ETS model. Therefore, this novel data-driven hybrid technique can be recommended as an effective and valuable tool in analyzing and estimating the temporal trends of the TB incidence in Tibet. Moreover, although the SARIMA model, NARNN model, ETS model, and SARIMA-NARNN mixture model produced a relatively low predictive performance relative to our proposed hybrid technique, they are also shown to have a good potential to model the TB epidemics because they gave a MAPE value less than $20 \%$ both in the mimic and predictive aspects, this is in good agreement with prior researches that used the above common models to analyze the temporal patterns of other infectious diseases (eg COVID-19, ${ }^{39}$ typhoid and paratyphoid fevers, ${ }^{40}$ schistosomiasis, ${ }^{41}$ and hand-foot-mouth disease $^{42}$ ). Also, our proposed hybrid model can be used to estimate the current intervention effects for $\mathrm{TB}$, if this model estimates a significantly higher incidence than the actual, meaning that the current interventions play an important role. Otherwise, additional or optimized measures are essential, such as optimization of the current tools, the pursuit of universal health coverage and social protection, the introduction of new vaccine and new prophylaxis, and the increase of the funding for $\mathrm{TB}{ }^{2}$

The SARIMA model has been recommended as a versatile tool for estimating and forecasting time series with marked seasonality and periodicity, and for a nonstationary time series, after differencing or making a transformation with logarithm or square root, this model can also be used and can achieve satisfactory forecasting. For instance, Chen et al developed a SARIMA method to estimate the epidemiological trends of the influenza incidence, they found that the $\operatorname{SARIMA}(0,1,0)$ $(0,1,2)_{12}$ method showed a MAPE value of $10.597 \%$ for predicting the influenza incidence in the urban area of Shenyang, and the $\operatorname{SARIMA}(1,1,1)(1,1,0)_{12}$ method had a MAPE value of $19.414 \%$ in the rural area. ${ }^{43}$ Gao et al observed that the $\operatorname{SARIMA}(0,1,7)(1,0,1)_{12}$ method displayed a good performance for forecasting the cumulative incidences of typhoid (MAPE $=13.257 \%$ ) and paratyphoid fevers (MAPE $=19.501 \%) .{ }^{40}$ Although the SARIMA model displays a good forecasting performance, it cannot describe the nonlinear information included in the TB morbidity in that this model assumes that there is a linear link between successive values of the time series. ${ }^{4}$ However, different from the SARIMA model, the dynamic NARNN method has been deemed as a promising alternative to address any nonlinear issue without any constraints because of its short-run memory function besides the common properties of static BPNN and GRNN. ${ }^{9,44,45}$ Thereby, researchers developed the traditional SARIMA-NARNN mixture model to take advantage of each method's potentials, and yet recent studies found that such a conventional combined mode may fail to model seasonal or trend variations effectively based on the unpreprocessed time series as the conventional model may not capture the linear and nonlinear components reasonably well, ${ }^{46}$ namely, the conventional hybrid models do not necessarily overmatch their individual predictions. ${ }^{19,46}$ Also, as evidenced by our and other work, the traditional SARIMA-NARNN or other conventional mixture models (such as SARIMA-BPNN and SARIMA-GRNN) cannot offer a performance improvement over the basic NARNN and ETS methods on the testing data. $^{19,47-49}$ For this reason, our proposed datadriven hybrid model is more reasonably customized for forecasting the different components via decomposing the target series into different linear and nonlinear traits based 

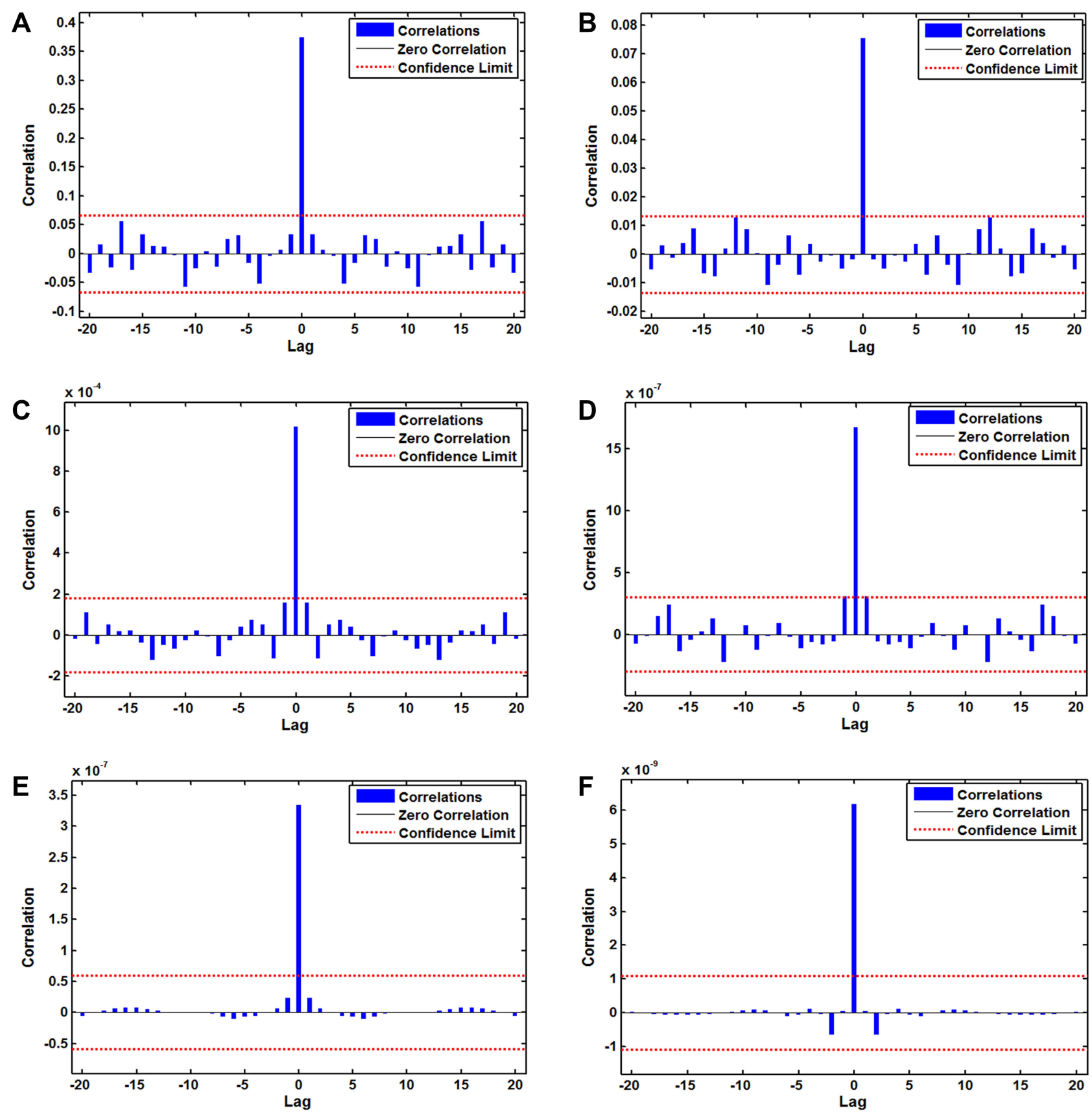

Figure 3 Estimated autocorrelogram of the residual sequence to forecast the six intrinsic mode functions (IMFs) decomposed by the EEMD method using the SARIMANARNN hybrid model. (A) IMFI, (B) IMF2, (C) IMF3, (D) IMF4, (E) IMF5, and (F) IMF6. For the estimated autocorrelations of the residual series from the different IMFs, all of them are inside of the $95 \mathrm{Cls}$ except for the one at lag zero, meaning that there is no correlated serial in the residuals of the best-fitting SARIMA-NARNN hybrid model. Thereby, there appears to be adequate and reasonable for forecasting upcoming epidemiological trends using these best-fitting models.

on the EEMD method. As expected, this hybrid technique is shown to be mechanically more robust and accurate for forecasting the TB incidence in Tibet compared with the others. Given its superiority and the low-cost data collection, this data-driven EEMD-SARIMA-NARNN combined technique seems to be transferable to analyze the epidemiological trends of the TB incidence in other regions or other types of infectious diseases, even of alltime series data, whereas much work is still required to investigate its efficiency and usefulness. Besides, of note, some new advanced deep mining methods (such as long short-term memory neural network (LSTM) and convolutional neural network (CNN)) have currently been applied to predict the dengue cases, ${ }^{50}$ daily land surface 
Table 5 Comparisons of the Fitted and Forecasted Accuracy Levels Among the Optimal Four Methods

\begin{tabular}{|c|c|c|c|c|c|c|c|c|c|c|}
\hline \multirow[t]{2}{*}{ Models } & \multicolumn{5}{|c|}{ Simulated Part } & \multicolumn{5}{|c|}{ Predicted Part } \\
\hline & MAD & MAPE & RMSE & MER & RMSPE & MAD & MAPE & RMSE & MER & RMSPE \\
\hline SARIMA model & 0.977 & 11.222 & 1.216 & 0.100 & 2.702 & 1.444 & 11.486 & 1.658 & 0.114 & 3.805 \\
\hline NARNN model & 0.770 & $12.30 \mid$ & 1.170 & 0.078 & 0.258 & 1.183 & 9.335 & 1.309 & 0.093 & 0.101 \\
\hline SARIMA-NARNN hybrid model & 0.648 & 8.634 & 0.856 & 0.066 & 0.155 & $\mathrm{I} .443$ & 11.281 & $\mathrm{I} .803$ & 0.114 & 0.143 \\
\hline EEMD-SARIMA-NARNN hybrid model & 0.438 & 6.814 & 0.669 & 0.045 & 0.141 & 0.965 & 7.958 & 1.133 & 0.076 & 0.096 \\
\hline \multicolumn{11}{|l|}{ Reduced percentage } \\
\hline$D$ vs $A$ & 55.169 & 39.280 & 44.984 & 55.000 & 94.782 & 33.172 & 30.716 & 31.665 & 33.333 & 97.477 \\
\hline$D$ vs $B$ & 43.117 & 44.606 & 42.821 & 42.308 & 45.349 & 18.428 & $|4.75|$ & 13.445 & 18.280 & 4.950 \\
\hline$D$ vs $C$ & 32.407 & 21.079 & 21.846 & 31.818 & 9.032 & 33.125 & 29.457 & 37.160 & 33.333 & 32.867 \\
\hline
\end{tabular}

Notes: A refers to the SARIMA method, B takes for the NARNN method, C signifies the traditional SARIMA-NARNN mixture method, D is the novel EEMD-SARIMANARNN mixture method.

Abbreviations: SARIMA, seasonal autoregressive integrated moving average method; EEMD, ensemble empirical mode decomposition; NARNN, nonlinear autoregressive artificial neural network; MAD, mean absolute deviation; MAPE, mean absolute percentage error; RMSE, root-mean-square error; MER, mean error rate; RMSPE, root-mean -square percentage error.

temperature, ${ }^{28}$ and ICD-9 codes of medical records, ${ }^{51}$ and these studies suggested that the LSTM and CNN also showed high accuracy for forecasting the target series. Therefore, further work is supposed to compare the forecasting ability between our proposed combined method and the above deep mining methods.

In this work, we discovered that the TB morbidity exhibited marked seasonal variations, predominantly peaking in late winter and early spring and a trough in autumn and early winter. Such a seasonal pattern is similar to previous findings from across China and most of the southwest regions of China (such as Qinghai, Xizang, and Yunnan), ${ }^{5,12,52}$ along with the results obtained in Japan and Spain. ${ }^{53,54}$ A preceding review to examine the seasonal variability based on 12 studies also supports further evidence that the peak patterns of TB are predominantly seen during the spring and summer seasons. ${ }^{55}$ And yet in disagreement with that in Eastern Cape, the United States, Korea, Mongolia, northern India, and Kuwait. ${ }^{9,56-58}$ In this study, an interesting finding is that the TB incidence series in Tibet displayed a primarily exclusive semi-annual seasonal pattern with longer highrisk seasons from January until August and low-risk seasons from September until December per year, which is inconsistent with those observed in earlier studies. ${ }^{5,12,52,55}$ Seemingly, various factors are related to the mechanisms underlying TB high-risk seasonal behavior. Firstly, Tibet, as a famous tourist city, attracts hundreds of thousands of persons every year, with tourists being over 40 million in 2019. ${ }^{59}$ Among them, the persons touring during the TB high-risk months approximately accounting for most of them. Large-scale population movements may drive the transmission of $\mathrm{TB}^{60}{ }^{60}$ which is also in keeping with the temporal patterns present in the high-risk months in the spatial-temporal sequence. Secondly, current studies have shown that meteorological parameters may play a pivotal role in the spread of TB. ${ }^{1,61}$ A study found that the TB incidence showed a decreased trend with the increases in temperature and precipitation in Qinghai. ${ }^{62}$ Another study suggested that wind speed is positively correlated with the TB incidence in Jinghong city of Yunnan. ${ }^{61}$ The climate of Tibet is characterized by low pressure, strong wind, heavy rainfall, and relatively low temperature (annual mean temperature is $8^{\circ} \mathrm{C}$ ), this climate type may increase the likelihood of contracting TB in the high-risk seasons as people tend to move indoors or crowded spaces for carrying out their activities and work. ${ }^{9}$ Thirdly, it is thought that vitamin D levels may be closely associated with the seasonal variation of TB, this is mediated through the elevated risk of activation of latent TB because of late winter nadirs in vitamin $\mathrm{D}$ levels. $^{52,56}$ The reduced sunshine in winter causes vitamin D deficiency, which further influences the potential of macrophages to kill the bacillus Mycobacterium tuberculosis in vitro. ${ }^{56,63}$ Fourthly, Lunar New Year, the foremost festival in China, may also play an important role in the seasonal peak patterns of TB since 

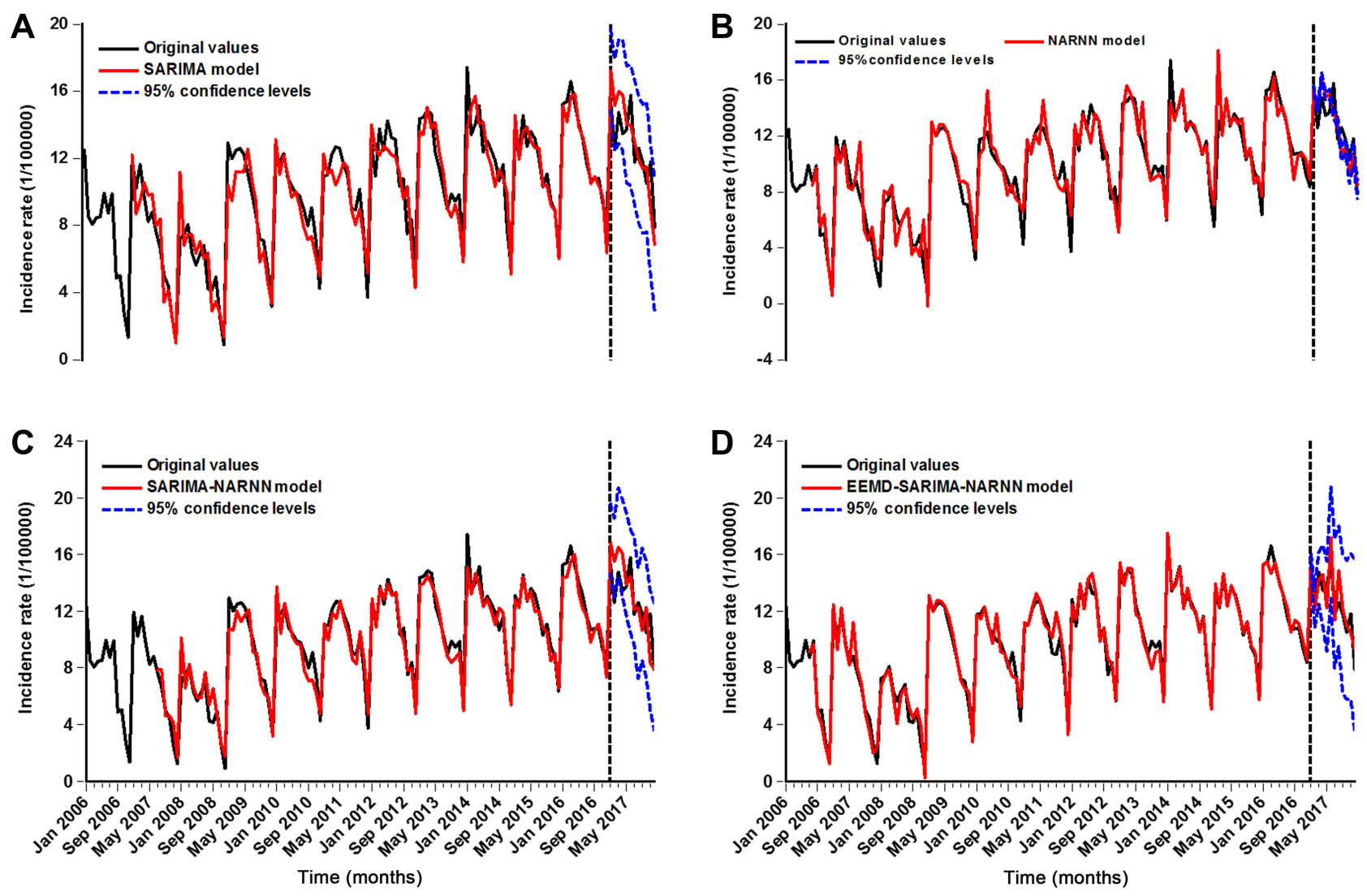

Figure 4 Time series plot showing the comparative results between original observations and fitted and predicted values using the selected best-fitting (A) SARIMA model, (B) NARNN model, (C) traditional SARIMA-NARNN hybrid model, and (D) novel EEMD-SARIMA-NARNN hybrid model (The curve at the left of the vertical black dotted line represents the fitted values, whereas the curve at the right denotes the forecasted values). As shown, the produced curve from the EEMD-SARIMA-NARNN hybrid model is closer to the actual both in the fitted and predicted aspects compared with the curves from the other models.

this festival is followed by the largest scale collective on the Tibet migrates, particularly in January and February per year. Given that there is an incubation of around $4-8$ weeks from infection to medical diagnosis, ${ }^{64}$ this is wholly in agreement with the maximum peak in March. Besides, it can be seen that there are lower reported cases in February than in January and March. This is derived from the fact that people do not tend to seek medical advice during the Lunar New Year period. Finally, in recent years, more and more activities involving the knowledge-attitude-behavior and new control modes of TB were implemented on World Tuberculosis Day in Tibet, which can enhance public awareness of TB and they are more willing to access healthcare. Besides, an investigation into other factors driving the seasonal variation should go on.

In contrast with the fact that the TB morbidity is falling per year at the rates of about $1.6 \%$ and $3 \%$ since 2005 in the world and China, respectively, ${ }^{2}$ the TB incidence was demonstrated to display a rapidly increasing trend in Tibet, with AAPC $=5.8$ from 2006 to 2017. To achieve the 2035 target of the End TB Strategy for $90 \%$ reductions in the TB incidence compared with 2015, the WHO estimates that the downtrend in the TB incidence requires to be accelerated at the rates of around $5 \%$ by $2020,10 \%$ by 2025, and $17 \%$ between 2025 and 2035 globally., ${ }^{2,65}$ The current epidemic patterns of TB in Tibet has been an extremely daunting challenge for reaching the aim of a world free of TB during different periods. ${ }^{65}$ Many reasons may be responsible for such an increasing trend in the TB epidemics in Tibet. Firstly, TB is an infectious disease of poverty; Tibet has poor essential health-care services and low socio-economic conditions, with the GDP ranking the last among 31 provinces and municipalities of China in $2019,{ }^{59}$ which may be the most important trigger for the rapid rise in the TB incidence. Secondly, local meteorological and environmental factors may also be important contributors. Thirdly, Buddhist monks and nuns that account for a significant proportion of the population in Tibet have poor health-care knowledge and they frequently 
undertake their religious activities in relatively small temples, irrespective of the time of the year, which facilitates the continued transmission among people. ${ }^{66}$ Fourthly, in recent years, the laboratory equipment and testing ability are continuously improved, and new techniques and methods of molecular biology detection and rapid diagnosis are popularized to improve the level of patient discovery in Tibet. Fifthly, around $80 \%$ of TB patients in Tibet had been resident in farming and pastoral areas, ${ }^{66}$ and many TB patients do not know that they can be examined and treated free of charge. Faced with the catastrophic costs for TB patients and their households, they often choose not to access healthcare, which further helps the spreading of TB among people. Finally, a study showed that the public awareness of core information on TB prevention and treatment was only $34 \%$, significantly lower than the national average level $(57.0 \%),{ }^{67}$ such a low public awareness may be a contributory factor in relation to the elevated $\mathrm{TB}$ incidence in Tibet. Besides, some other reasons (such as alcohol use disorders, diabetes, HIV infection, smoking, and undernourishment) may also play a role. Confronted with such a worsening tendency, some additional or comprehensive intervention strategies must be taken to combat TB in Tibet.

The present study was subject to some potential weaknesses. Firstly, the time series of the TB incidence was derived from a passive monitoring system, and therefore the underreporting may be inevitable. Secondly, we only used a dataset spanning 12 years to construct the models and some detailed information (such as sex and age) of the TB cases cannot be extracted, which limits further sensitivity analysis for our model. Thirdly, there is currently still in the absence of a guidance to determine the suitable key parameters of ANNs (such as hidden units and feedback delays). In applications, a series of experiments need to be performed by trial and error until the best performance indices are detected. Fourthly, incorporating potential influencing factors of TB into the EEMD-SARIMANARNN method may favor the improvement of the forecasting performance, and yet this failed to be done in our work. Fifthly, whether this novel mixture method can also achieve a high-accuracy prediction for other infectious diseases or time series, further research should be undertaken to explore its potential use. Finally, in applications, the newly reported TB cases should be added into the model in time to make sure of its forecasting accuracy level.

\section{Conclusions}

Our proposed novel data-driven EEMD-SARIMA-NARNN hybrid method can better capture the dynamic dependence characteristics of the TB incidence in Tibet compared with the basic SARIMA model, basic NARNN model, ETS model, and traditional SARIMA-NARNN combined method, which can be recommended as a helpful and instrumental policy-supportive tool for planning the healthcare resources ahead of time and directing the intensity and type of current interventions required to end the TB epidemic. Besides, considering the ongoing increases in TB cases, some additional or comprehensive intervention strategies must be taken to combat TB in Tibet.

\section{Data Sharing Statement}

All the data supporting the findings of the work are contained within the study.

\section{Acknowledgments}

We appreciate the Chinese CDC for providing the monthly number of TB cases. This project was supported by the Innovation and Entrepreneurship Training Project for University Students of Henan Province (S202010472007) and Xinxiang Medical University (XYXSKYZ201932) and the Key Scientific Research Project of Universities in Henan (21A330004).

\section{Author Contributions}

All authors made substantial contributions to conception and design, acquisition of data, or analysis and interpretation of data; took part in drafting the article or revising it critically for important intellectual content; agreed to submit to the current journal; gave final approval of the version to be published; and agree to be accountable for all aspects of the work.

\section{Disclosure}

The authors report no conflicts of interest in this work.

\section{References}

1. Li ZQ, Pan HQ, Liu Q, Song H, Wang JM. Comparing the performance of time series models with or without meteorological factors in predicting incident pulmonary tuberculosis in eastern China. Infect Dis Poverty. 2020;9(1):151. doi:10.1186/s40249-020-00771-7

2. WHO. Global tuberculosis report 2020. Available from: https://apps. who.int/iris/handle/10665/336069. Accessed December 25, 2020.

3. Ding C, Wang S, Shangguan Y, et al. Epidemic trends of tuberculosis in China from 1990 to 2017: evidence from the global Burden of Disease Study. Infect Drug Resist. 2020;13:1663-1672. doi:10.2147/ idr.s249698 
4. Wang Y, Xu C, Ren J, et al. Secular seasonality and trend forecasting of tuberculosis incidence rate in China using the advanced error-trend-seasonal framework. Infect Drug Resist. 2020;13:733-747. doi:10.2147/IDR.S238225

5. Wang Y, Xu C, Zhang S, et al. Temporal trends analysis of tuberculosis morbidity in mainland China from 1997 to 2025 using a new SARIMA-NARNNX hybrid model. BMJ Open. 2019;9(7):e024409. doi:10.1136/bmjopen-2018-024409

6. Li Z, Wang Z, Song H, et al. Application of a hybrid model in predicting the incidence of tuberculosis in a Chinese population. Infect Drug Resist. 2019;12:1011-1020. doi:10.2147/idr.s190418

7. Zhang YQ, Li XX, Li WB, et al. Analysis and predication of tuberculosis registration rates in Henan Province, China: an exponential smoothing model study. Infect Dis Poverty. 2020;9(1):123. doi:10.1186/s40249-020-00742-y

8. Wang YW, Shen ZZ, Jiang Y. Comparison of autoregressive integrated moving average model and generalised regression neural network model for prediction of haemorrhagic fever with renal syndrome in China: a time-series study. BMJ Open. 2019;9(6): e025773. doi:10.1136/bmjopen-2018-025773

9. Adeboye A, Davies O, Akinwumi O, James N, Ruffin M. Seasonality and trend forecasting of tuberculosis prevalence data in Eastern Cape, South Africa, using a hybrid model. Int J Environ Res Public Health. 2016;13(8):757. doi:10.3390/ijerph13080757

10. Zhang X, Zhang T, Young AA, Li X. Applications and comparisons of four time series models in epidemiological surveillance data. PLoS One. 2014;9(2):e88075. doi:10.1371/journal.pone.0088075

11. Wu W, An SY, Guan P, Huang DS, Zhou BS. Time series analysis of human brucellosis in mainland China by using Elman and Jordan recurrent neural networks. BMC Infect Dis. 2019;19(1):414. doi:10.1186/s12879-019-4028-X

12. Wang Y, Xu C, Li Y, et al. An advanced data-driven hybrid model of SARIMA-NNNAR for tuberculosis incidence time series forecasting in Qinghai Province, China. Infect Drug Resist. 2020;13:867-880. doi:10.2147/IDR.S232854

13. Tian CW, Wang H, Luo XM. Time-series modelling and forecasting of hand, foot and mouth disease cases in China from 2008 to 2018. Epidemiol Infect. 2019;147:e82. doi:10.1017/S095026881800362X

14. Weng RX, Fu HL, Zhang CL, et al. Time series analysis and forecasting of chlamydia trachomatis incidence using surveillance data from 2008 to 2019 in Shenzhen, China. Epidemiol Infect. 2020;148: e76. doi:10.1017/s0950268820000680

15. Qiu H, Zeng D, Yi J, et al. Forecasting the incidence of acute haemorrhagic conjunctivitis in Chongqing: a time series analysis. Epidemiol Infect. 2020;148:e193. doi:10.1017/S095026882000182X

16. Wang KW, Deng C, Li JP, Zhang YY, Li XY, Wu MC. Hybrid methodology for tuberculosis incidence time-series forecasting based on ARIMA and a NAR neural network. Epidemiol Infect. 2017;145(6):1118-1129. doi:10.1017/S0950268816003216

17. Pan A, Liu L, Wang C, et al. Association of public health interventions with the epidemiology of the COVID-19 outbreak in Wuhan, China. JAMA. 2020;323(19):1915. doi:10.1001/jama.2020.6130

18. Lin YJ, Lin HC, Yang YF, et al. Association between ambient air pollution and elevated risk of tuberculosis development. Infect Drug Resist. 2019;12:3835-3847. doi:10.2147/IDR.S227823

19. Taskaya-Temizel T, Casey MC. A comparative study of autoregressive neural network hybrids. Neural Net. 2005;18(5-6):781-789. doi:10.1016/j.neunet.2005.06.003

20. Zhang X, Zhang Q, Zhang G, Nie Z, Gui Z, Hybrid A. Model for annual runoff time series forecasting using Elman neural network with ensemble empirical mode decomposition. Water-Sui. 2018;10 (4):416. doi:10.3390/w10040416

21. Wang H, Tian CW, Wang WM, Luo XM. Time-series analysis of tuberculosis from 2005 to 2017 in China. Epidemiol Infect. 2018;146 (8):935-939. doi:10.1017/S0950268818001115
22. Deb M, Chakrabarty TK. A wavelet based hybrid SARIMA-ETS model to forecast electricity consumption. Elect J Appl Statistical Anal. 2017;10(2):408-430.

23. Wu Z, Huang NE. Ensemble empirical mode decomposition: a noise-assisted data analysis method. Adv Adapt Data Anal. 2009;01 (01):1-41. doi:10.1142/s1793536909000047

24. Huang NE, Shen Z, Long SR. A new view of nonlinear water waves: the Hilbert spectrum. Annu Rev Fluid Mech. 1999;31 (1):417-457.

25. Zuo Z, Wang M, Cui H, et al. Spatiotemporal characteristics and the epidemiology of tuberculosis in China from 2004 to 2017 by the nationwide surveillance system. BMC Public Health. 2020;20 (1):1284. doi:10.1186/s12889-020-09331-y

26. Bartholomew D, Box GEP, Jenkins GM. Time Series Analysis: Forecasting and Control. 5th ed. Hoboken, New Jersey: John Wiley and Sons Inc.; 2015:14

27. Qiu X, Ren Y, Suganthan PN, Amaratunga GAJ. Empirical mode decomposition based ensemble deep learning for load demand time series forecasting. Appl Soft Comput. 2017;54:246-255.

28. Zhang X, Zhang Q, Zhang G, et al. Data-driven model for daily land surface temperature forecasting using long short-term memory neural network based on ensemble empirical mode decomposition. Int J Environ Res Public Health. 2018;15(5):1032. doi:10.3390/ ijerph15051032

29. Zhou Q, Jiang H, Wang J, Zhou J. A hybrid model for PM2.5 forecasting based on ensemble empirical mode decomposition and a general regression neural network. Sci Total Environ. 2014;496:264-274. doi:10.1016/j.scitotenv.2014.07.051

30. Jian Z, Yan R, Gao RX, Feng Z. Performance enhancement of ensemble empirical mode decomposition. Mech Syst Signal Process. 2010;24(7):P.2104-2123.

31. Wang $\mathrm{W}$, Chau $\mathrm{K}, \mathrm{Xu} \mathrm{D}$, Chen $\mathrm{X}$. Improving forecasting accuracy of annual runoff time series using ARIMA based on EEMD decomposition. Water Resources Manage. 2015;29(8):2655-2675.

32. Wang Y, Xu C, Wu W, et al. Time series analysis of temporal trends in hemorrhagic fever with renal syndrome morbidity rate in China from 2005 to 2019. Sci Rep. 2020;10(1):9609. doi:10.1038/s41598020-66758-4

33. Liu Q, Li Z, Ji Y, et al. Forecasting the seasonality and trend of pulmonary tuberculosis in Jiangsu Province of China using advanced statistical time-series analyses. Infect Drug Resist. 2019;12:2311-2322. doi:10.2147/idr.s207809

34. Lam KC, Oshodi OS. Forecasting construction output: a comparison of artificial neural network and Box-Jenkins model. Eng Construction Arch Manage. 2016;23(3):302-322.

35. Maleki A, Nasseri S, Aminabad MS, Hadi M. Comparison of ARIMA and NNAR models for forecasting water treatment plant's influent characteristics. Ksce J Civil Eng. 2018;(6):1-13.

36. Thoplan R. Simple v/s sophisticated methods of forecasting for Mauritius monthly tourist arrival data. Int J Stat Appl. 2014;4 (5):217-223. doi:10.5923/j.statistics.20140405.01

37. Zhou L, Jing X, Yu L, et al. Using a hybrid model to forecast the prevalence of schistosomiasis in humans. Int $J$ Environ Res Public Health. 2016;13(4):355. doi:10.3390/ijerph13040355

38. Pao HT. Forecasting energy consumption in Taiwan using hybrid nonlinear models. Energy. 2009;34(10):1438-1446. doi:10.1016/j. energy.2009.04.026

39. Saba AI, Elsheikh AH. Forecasting the prevalence of COVID-19 outbreak in Egypt using nonlinear autoregressive artificial neural networks. Process Saf Environ Protection. 2020;141:1-8. doi:10.1016/j.psep.2020.05.029

40. Gao J, Li J, Wang M. Time series analysis of cumulative incidences of typhoid and paratyphoid fevers in China using both Grey and SARIMA models. PLoS One. 2020;15(10):e0241217. doi:10.1371/ journal.pone. 0241217 
41. Yu XY, Chen Z, Qi LX. Comparative study of SARIMA and NARX models in predicting the incidence of schistosomiasis in China. Mathematical Biosci Eng. 2019;16(4):2266-2276. doi:10.3934/ mbe. 2019112

42. Yu L, Zhou L, Tan L, et al. Application of a new hybrid model with seasonal auto-regressive integrated moving average (ARIMA) and nonlinear auto-regressive neural network (NARNN) in forecasting incidence cases of HFMD in Shenzhen, China. PLoS One. 2014;9 (6):e98241. doi:10.1371/journal.pone.0098241

43. Chen Y, Leng K, Lu Y, et al. Epidemiological features and time-series analysis of influenza incidence in urban and rural areas of Shenyang, China, 2010-2018. Epidemiol Infect. 2020;148:e29. doi:10.1017/ S0950268820000151

44. Wang Y, Xu C, Zhang S, et al. Development and evaluation of a deep learning approach for modeling seasonality and trends in hand-footmouth disease incidence in mainland China. Sci Rep. 2019;9(1):8046. doi:10.1038/s41598-019-44469-9

45. Nury AH, Hasan K, Alam MJB. Comparative study of wavelet-ARIMA and wavelet-ANN models for temperature time series data in northeastern Bangladesh. J King Saud Univ. 2017;29 (1):47-61.

46. Qi ZM. Neural network forecasting for seasonal and trend time series. Eur J Oper Res. 2005;501-514.

47. Zhou L, Zhao P, Wu D, Cheng C, Huang H. Time series model for forecasting the number of new admission inpatients. BMC Med Inform Decis Mak. 2018;18(1):39. doi:10.1186/s12911-018-0616-8

48. Wei W, Jiang J, Gao L, et al. A new hybrid model using an autoregressive integrated moving average and a generalized regression neural network for the incidence of tuberculosis in Heng County, China. Am J Trop Med Hyg. 2017;97(3):799-805. doi:10.4269/ ajtmh.16-0648

49. Wu W, Guo J, An S, et al. Comparison of two hybrid models for forecasting the incidence of hemorrhagic fever with renal syndrome in Jiangsu Province, China. PLoS One. 2015;10(8):e0135492. doi:10.1371/journal.pone.0135492

50. Xu J, Xu K, Li Z, et al. Forecast of dengue cases in 20 Chinese cities based on the deep learning method. Int J Environ Res Public Health. 2020;17:2. doi:10.3390/ijerph17020453

51. Hsu JL, Hsu TJ, Hsieh CH, Singaravelan A. Applying convolutional neural networks to predict the ICD-9 codes of medical records. Sensors (Basel, Switzerland). 2020;20:24. doi:10.3390/s20247116

52. Wubuli A, Li Y, Xue F, Yao X, Upur H, Wushouer Q. Seasonality of active tuberculosis notification from 2005 to 2014 in Xinjiang, China. PLoS One. 2017;12(7):e0180226. doi:10.1371/journal.pone.0180226

53. Sumi A, Kobayashi N. Time-series analysis of geographically specific monthly number of newly registered cases of active tuberculosis in Japan. PLoS One. 2019;14(3):e0213856. doi:10.1371/journal. pone. 0213856
54. Rios M, Garcia JM, Sanchez JA, Perez D. A statistical analysis of the seasonality in pulmonary tuberculosis. Eur J Epidemiol. 2000;16 (5):483-488. doi:10.1023/a:1007653329972

55. Fares A. Seasonality of tuberculosis. J Glob Infect Dis. 2011;3 (1):46-55. doi:10.4103/0974-777X.77296

56. Willis MD, Winston CA, Heilig CM, Cain KP, Walter ND, Mac Kenzie WR. Seasonality of tuberculosis in the United States, 1993-2008. Clin Infect Dis. 2012;54(11):1553-1560. doi:10.1093/ cid $/$ cis 235

57. Kim EH, Bae JM. Seasonality of tuberculosis in the Republic of Korea, 2006-2016. Epidemiol Health. 2018;40:e2018051. doi:10.4178/epih.e2018051

58. Thorpe LE, Frieden TR, Laserson KF, Wells C, Khatri GR. Seasonality of tuberculosis in India: is it real and what does it tell us? Lancet. 2004;364(9445):1613-1614. doi:10.1016/s0140-6736(04) 17316-9

59. Tibet Autonomous Region Department of Tourism Development. Available from: http://lyfzt.xizang.gov.cn/. Accessed December 25, 2020.

60. Li X, Li T, Tan S. Males, ages $\geq 45$ years, businessperson, floating population, and rural residents may be considered high-risk groups for tuberculosis infection in Guangzhou, China: a review of 136,394 tb confirmed cases. Rev Inst Med Trop Sao Paulo. 2013;55 (5):366-368. doi:10.1590/s0036-46652013000500013

61. Xiao Y, He L, Chen Y, et al. The influence of meteorological factors on tuberculosis incidence in Southwest China from 2006 to 2015. Sci Rep. 2018;8(1):10053. doi:10.1038/s41598-018-28426-6

62. Rao HX, Zhang X, Zhao L, et al. Spatial transmission and meteorological determinants of tuberculosis incidence in Qinghai Province, China: a spatial clustering panel analysis. Infect Dis Poverty. 2016;5 (1):45. doi:10.1186/s40249-016-0139-4

63. Ganmaa D, Uyanga B, Zhou X, et al. Vitamin D supplements for prevention of tuberculosis infection and disease. $N$ Engl J Med. 2020;383(4):359-368. doi:10.1056/NEJMoa1915176

64. Rao H, Shi X, Zhang X. Using the Kulldorff's scan statistical analysis to detect spatio-temporal clusters of tuberculosis in Qinghai Province, China, 2009-2016. BMC Infect Dis. 2017;17(1):578. doi:10.1186/s12879-017-2643-y

65. WHO. The End TB Strategy. 2014. Available from: https:// wwwwhoint/tb/End_TB_brochurepdf. Accessed December 25, 2020.

66. Bureau of statistics of Tibet Autonomous Region. Statistical Yearbook of Tibet 2020. Available from:http://tjj.xizang.gov.cn/. Accessed December 25, 2020.

67. Guo J, Wang J. Sorangza. A survey of the knowledge of core information on tuberculosis prevention and control in Tibet Autonomous Region. World Latest Med Inf. 2020;19(73):217-219.
Infection and Drug Resistance

\section{Publish your work in this journal}

Infection and Drug Resistance is an international, peer-reviewed openaccess journal that focuses on the optimal treatment of infection (bacterial, fungal and viral) and the development and institution of preventive strategies to minimize the development and spread of resistance. The journal is specifically concerned with the epidemiology of antibiotic resistance and the mechanisms of resistance development and diffusion in both hospitals and the community. The manuscript management system is completely online and includes a very quick and fair peerreview system, which is all easy to use. Visit http://www.dovepress.com/ testimonials.php to read real quotes from published authors 